\title{
The Unusual Evolutionary State of Nova Scorpii 1994
}

\author{
Christopher A. Tout, Enikő Regős \\ Institute of Astronomy, The Observatories, Madingley Road, Cambridge \\ CB3 OHA, England \\ Dayal Wickramasinghe \\ Department of Mathematics, Australian National University, Canberra, \\ ACT 0200, Australia
}

\begin{abstract}
We have investigated possible models for the evolutionary status of the black-hole soft X-ray transient Nova Scorpii. We propose that it is a main-sequence star in which convective overshooting, or some equivalent mixing, occurs at the core-envelope boundary. Recent detections of chemical pollution on the surface of the secondary suggest that the black hole formed in a supernova whose ejecta left and fell back at a sufficiently low velocity to accrete on to the secondary. We examine the effects of increased opacity, increased metallicity and reduced hydrogen abundance on the evolution of the secondary in the hope that these might lead to an alternative scenario. All these only exacerbate the problem.
\end{abstract}

\section{Introduction}

The transient X-ray binary Nova Sco 1994 consists of a black hole (the primary) of mass $7.02 \pm 0.22 M_{\odot}$ (Orosz \& Bailyn 1997) accreting material from an accretion disc which is fed by its evolved companion (the secondary) of mass $2.34 \pm 0.12 M_{\odot}$. The spectral classification, F3 - F5 puts such a star in the middle of the Hertzsprung gap where finding it is rather unusual. A $2.34 M_{\odot}$ star spends about $3 \times 10^{7} \mathrm{yr}$ in the Hertzsprung gap, compared to $6 \times 10^{8} \mathrm{yr}$ on the main sequence and about $2.5 \times 10^{8} \mathrm{yr}$ in its subsequent evolution. However, its radius increases by a factor of two, about the same as when crossing the main sequence so that it is not so unlikely for short orbital period systems to first fill their Roche lobes during this evolutionary phase.

van Paradijs (1996) found that the average accretion rate on to the black hole has been less than $1.26 \times 10^{-10} M_{\odot} \mathrm{yr}^{-1}$. However, as pointed out by Kolb et al. (1997), the secondary star should be expanding on a thermal timescale of about $10^{7} \mathrm{yr}$ and hence transferring matter at a rate of about $10^{-7} M_{\odot} \mathrm{yr}^{-1}$.

\section{Roche-filling models}

The solid track in Fig. 1 is the evolution of a $2.34 M_{\odot}$ star in the HertzsprungRussell diagram which passes close to the filled circle that marks the position of 


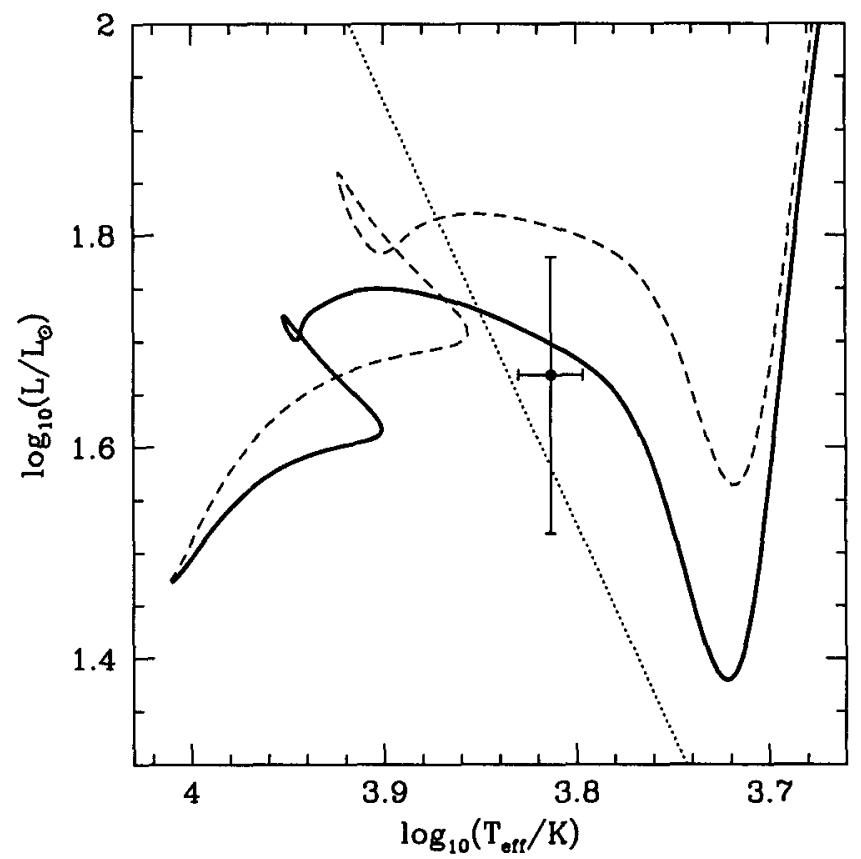

Figure 1. The track of a $2.34 M_{\odot}$ star. The solid track is for a star with no convective overshooting while the dashed line has overshooting. The observed position of the secondary star in GRO J1655-40 is shown as a point with error bars and the locus of blackbodies with a radius equal to its Roche-lobe radius appears as a straight dotted line.

the secondary star. The dotted line is the size of the Roche lobe of the secondary calculated from its mass and the period of $2.62157 \pm 0.00015 \mathrm{~d}$ and is independent of the mass of the black hole. This gives an upper limit to the size of the star of $4.88 \pm 0.10 R_{\odot}$ while the actual radius determined from the effective temperature of $6500 \pm 250 \mathrm{~K}$ and luminosity of $46.6 \pm 13.6 L_{\odot}$ is $5.4 \pm 1.2 R_{\odot}$. The proximity of these radii and the existence of an X-ray emitting disc suggest that this star is filling its Roche lobe.

Fig. 2 shows the track of a star that fills its Roche lobe soon after reaching the Hertzsprung gap and has reached a mass of about $2.34 M_{\odot}$ by the time it reaches the vicinity of Nova Sco 1994. This system began with $M_{1}=6.86 M_{\odot}$, $M_{2}=2.5 M_{\odot}$ and $P_{\text {orb }}=2.23 \mathrm{~d}$. Under the assumption of conservative mass transfer, conservation of total mass and orbital angular momentum, $\dot{M}_{1}=-\dot{M}_{2}$ and $\dot{J}_{\mathrm{orb}}=0$, it has the masses and period of Nova Sco 1994 once it has transferred $0.16 M_{\odot}$. Plotted in the same figure, as a dotted line, is the corresponding mass transfer-rate. The point at which the star first fills its Roche lobe is easily identified at about $\log _{10}\left(T_{\text {eff }} / K\right)=3.9$ soon after the end of the main sequence. The mass-transfer rate rapidly exceeds $10^{-8} M_{\odot} \mathrm{yr}^{-1}$ rising to well above $10^{-7} M_{\odot} \mathrm{yr}^{-1}$ for most of the Hertzsprung-gap evolution. 


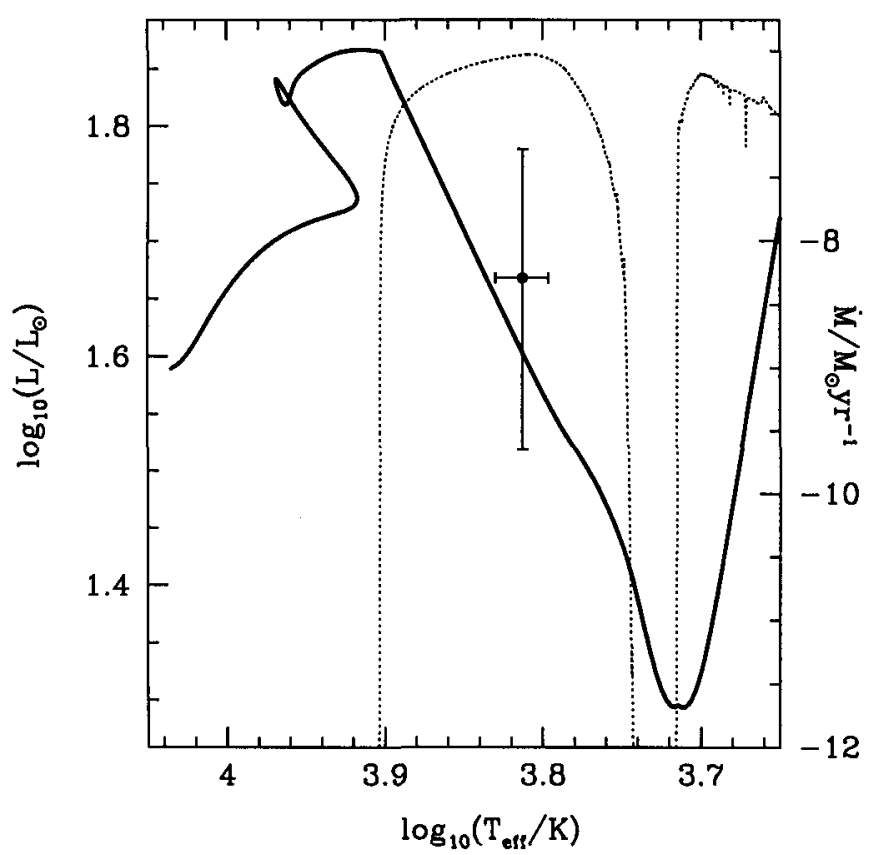

Figure 2. The track of a $2.5 M_{\odot}$ star in a binary and which is forced to transfer mass to its companion when it fills its Roche lobe which occurs while crossing the Hertzsprung gap. The dotted line and righthand scale give the mass-transfer rate as a function of temperature.

\section{Non-Roche-filling models}

The secondary is beginning to develop a convective envelope, though this is still of very little mass at the observed temperature, and so we might expect a stellar wind similar to that of the Sun and red giants to be leaving its surface. The black hole is well situated to accrete a substantial fraction of this stellar wind and we investigate whether this is the route taken by the accreted material. A Reimers mass-loss rate with Bondi-Hoyle accretion gives $2 \times 10^{-11} M_{\odot} \mathrm{yr}^{-1}$ to be compared with the upper limit of $1.26 \times 10^{-10} M_{\odot} \mathrm{yr}^{-1}$ of van Paradijs. However, because of the secondary's thin convective envelope, we might expect a substantially weaker wind or possibly none at all.

\section{Main-sequence models with overshooting}

Stars of $1.3 M_{\odot}$ or more have a convective core on the main sequence, because the CNO cycle dominates hydrogen fusion. In mixing length theories of convection cells accelerate right up to the boundary of the convective region given by the Schwarzschild criterion. Such cells would then continue to move across the boundary until their velocity is reduced to zero by the decelerating forces in 


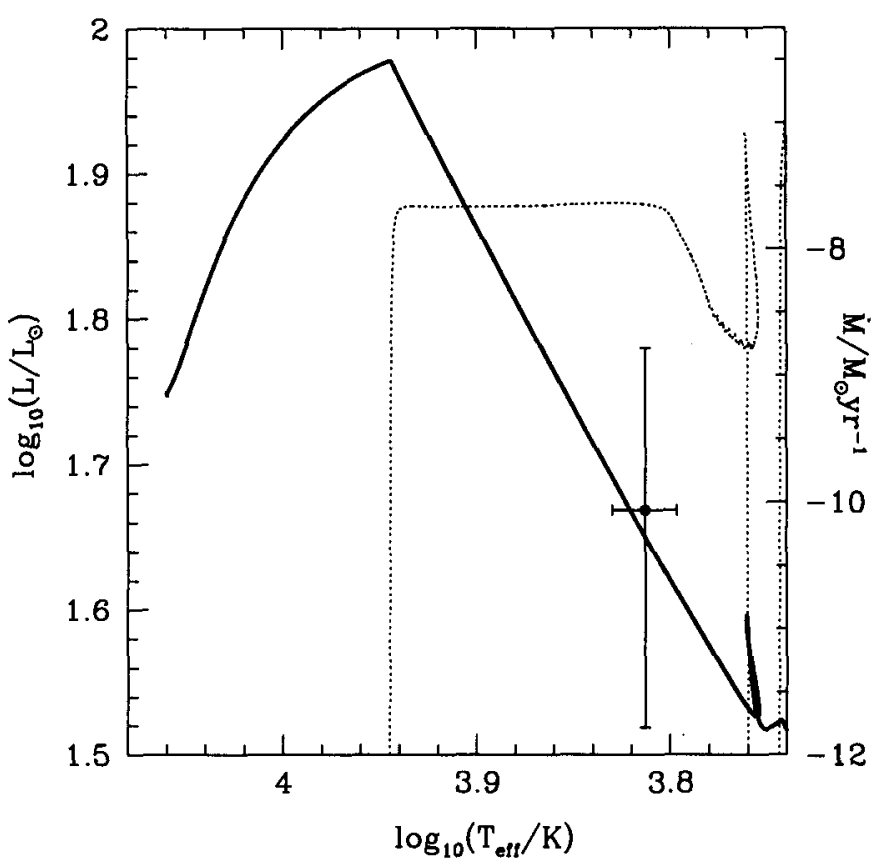

Figure 3. Similar to Fig. 2 but for a $2.75 M_{\odot}$ secondary star with convective overshooting that fills it Roche lobe on the main sequence.

the stable radiative region. This overshooting at the edge of the convective core mixes in extra hydrogen fuel that prolongs the main-sequence allowing the star to reach somewhat larger radii before the evolution accelerates in the Hertzsprung gap. The dashed track in Fig. 1 is a $2.34 M_{\odot}$ star with overshooting. Such progenitors of Nova Sco can grow large enough to fill their Roche lobes while still on the main sequence. Fig. 3 shows the evolution of a system that began with $M_{2}=2.75 M_{\odot}, M_{1}=6.61 M_{\odot}$ and $P_{\text {orb }}=1.935 \mathrm{~d}$. The secondary star fills its Roche lobe before the hook at the end of the main sequence and evolves right through the observational error box as mass is transferred to the black hole. Similar models with secondaries of lower mass are also satisfactory.

\section{Contamination}

Israelian et al. (1999) found increased metal (particularly $\mathrm{O}, \mathrm{Mg}, \mathrm{Si}, \mathrm{S}$ and $\mathrm{Ti}$ ) abundances by factors of about ten over solar on the surface of the secondary. These are probably the result of contamination by the inner parts of the star that became the black hole. Though of great interest, we shall not discuss the formation mechanism here but shall assume sufficient ejecta was accreted by the secondary and that thermohaline mixing led to a uniform composition before any 


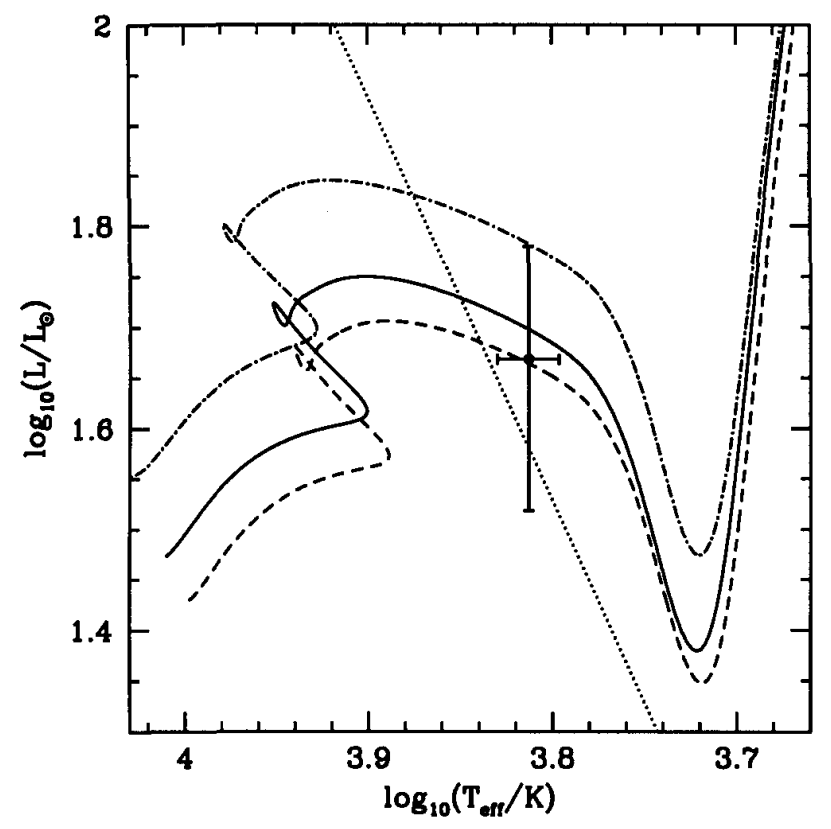

Figure 4. The effects of contamination on the evolution of the secondary star. The dashed track is with increased opacity while the dot-dashed track has decreased hydrogen abundance too.

significant evolution. This contamination can affect the structure and evolution of the secondary in three ways:

1) Increase in opacity (dashed track in Fig. 4)

2) Decrease in hydrogen abundance (dot-dashed)

3) Increase in CNO elements and nuclear reaction rates (as dot-dashed)

Fig. 4 shows the standard evolution of the non-interacting star of $2.34 M_{\odot}$ together with tracks for which effects 1) and 2) have been added. The third effect is negligible, only dropping the central temperature slightly to maintain the CNO burning rate. In each case the star is more evolved by the time it reaches its Roche lobe and so the problems are only exacerbated.

\section{Conclusions}

Our first model, a secondary star evolving across the Hertzsprung gap and filling its Roche lobe, can be ruled out because the expected mass-transfer rate is over 1,000 times the observed upper limit. The second, a secondary star in the Hertzsprung gap, not filling its Roche lobe but transferring mass via a stellar wind is acceptable but unsatisfactory because no deep convective envelope has developed at the observed temperature. The third, a main-sequence secondary 
with convective overshooting, can be made to fit with a transfer rate only 16 times van Paradijs' estimate and is our favoured model. Indeed Pols et al. (1997) demonstrate that convective overshooting, or some equivalent mixing at the core-envelope boundary, does take place in main-sequence stars with masses between 2 and $3 M_{\odot}$.

An alternative model of the light curves (Beer \& Podsiadlowski 2001) suggests much lower masses of $5.4 M_{\odot}$ for the black hole and $1.45 M_{\odot}$ for its companion. If correct these masses allow a model in which the secondary evolves normally to fill its Roche lobe near the end of the main sequence or in its more restrained Hertzsprung gap. In addition Orosz et al. (2001 private communication) have found evidence for contamination of the secondary star in V4641 Sgr.

Thus Nova Sco 1994 may be losing its bizarre claims! But for the moment, at least, it remains the most easily observed soft X-ray transient with a measured black hole component and so it will continue to stand out from the crowd for a while.

Acknowledgments. CAT thanks Churchill College, Cambridge for a Fellowship.

\section{References}

Beer, M. E., \& Podsiadlowski, P. 2001, in ASP Conf. Ser. Vol. 229, Evolution of Binary and Multiple Star Systems, ed. P. Podsiadlowski, S. Rappaport, A. R. King, F. D'Antona, \& L. Burderi (San Francisco: ASP), 547

Kolb, U., King, A. R., Ritter, H., \& Frank, J. 1997, ApJ, 485, L33

Orosz, J. A., \& Bailyn, C. D. 1997, ApJ, 477, 876

Pols, O. R., Tout, C. A., Schröder, K-P., Eggleton, P. P., \& Manners, J. 1997, MNRAS, 289, 869

van der Hooft, F., Heemskerk, M. H. M., Alberts, F., \& van Paradijs, J. 1998, A\&A, 329, 538

van Paradijs, J. 1996, ApJ, 464, L139 